\title{
Attended versus unattended blood pressure measurement in real-life settings in patients with chronic kidney disease
}

\author{
KATARZYNA PĘCZEK ${ }^{A-G}$, PIOTR PĘCZEK ${ }^{B, C, E, F}$, MICHA NOWICKI ${ }^{\mathrm{C}-\mathrm{G}}$ \\ ORCID ID: 0000-0001-6102-854X \\ Department of Nephrology, Hypertension and Kidney Transplantation, Medical University of Lodz, Poland
}

A - Study Design, B - Data Collection, C - Statistical Analysis, D - Data Interpretation, E - Manuscript Preparation, F - Literature Search, G - Funds Collection

Summary Background. Systolic Blood Pressure Intervention Trial (SPRINT) has raised interest in unattended automated office blood pressure (BP) measurement. It remains to be determined whether unattended BP measurement may yield lower values than conventional attended measurements in patients with very high cardiovascular risk, e.g. with chronic kidney disease (CKD).

Objectives. The aim of the study was to investigate the differences in attended (ABP) vs unattended BP (NABP) in hospitalised patients with CKD.

Material and methods. 60 patients were included (33 M, $27 \mathrm{~F}$; age $65.6 \pm 14.0$ years, eGFR $41.6 \pm 28.5(5.2-94.4 \mathrm{ml} / \mathrm{min}))$. BP (blood pressure) was first measured using the conventional auscultatory method by a medical staff member, and after a five-minute rest, three additional automated measurements with OMRON M10-IT were taken without the presence of medical staff. The same procedure was repeated over two consecutive days without any modification of antihypertensive treatment.

Results. Mean unattended systolic blood pressure (NASBP) and unattended diastolic blood pressure (NADBP) were 143.6 and $77.9 \mathrm{~mm}$ $\mathrm{Hg}$, respectively. The respective values of attended blood pressure (ABP) were 150.8 and $81.4 \mathrm{~mm} \mathrm{Hg}$. All ABP values were significantly higher than unattended blood pressure values $(p=0.009, p=0.04$ and $p=0.01$, respectively). The differences between ABP and NABP did not correlate with eGFR. The difference between ABP and NABP was similar in diabetic and non-diabetic patients, as well as smokers vs non-smokers.

Conclusions. Attended BP is significantly higher than unattended BP in patients with CKD regardless of eGFR. Automated BP measurement could become routine practice in patients with CKD.

Key words: hypertension, blood pressure, renal insufficiency chronic.

Pęczek K, Pęczek P, Nowicki M. Attended versus unattended blood pressure measurement in real-life settings in patients with chronic kidney disease. Fam Med Prim Care Rev 2019; 21(4): 360-363, doi: https://doi.org/10.5114/fmpcr.2019.90168.

\section{Background}

The European Society of Cardiology (ESC)/European Society of Hypertension (ESH) and the American Heart Association (AHA)/American College of Cardiology (ACC) recommend different target blood pressure values for patients with arterial hypertension. While the target blood pressure recommended by ESC/ /ESH is less than $130 / 80 \mathrm{~mm} \mathrm{Hg}$ in patients under 65 years of age and less than $140 / 80 \mathrm{~mm} \mathrm{Hg}$ in patients over 65 years of age [1], AHA/ACC guidelines recommend values lower than 130/80 $\mathrm{mm} \mathrm{Hg}$ regardless of age [2]. These disparities have been largely influenced by a different interpretation of the results of the seminal Systolic Blood Pressure Intervention Trial (SPRINT) [3].

SPRINT was undoubtedly the most controversial study affecting the diagnostics and management of arterial hypertension. The trial results may also have implications for the management of high BP in patients with chronic kidney disease (CKD), since $28 \%$ of the SPRINT population had been previously diagnosed with chronic kidney disease with an estimated glomerular filtration rate (eGFR) $20-59 \mathrm{ml} / \mathrm{min} / 1.73 \mathrm{~m}^{2}$. In SPRINT, the treatment effects did not differ between the patients with or without chronic kidney disease; however, the intensive-treatment CKD subgroup showed a lower incidence of all-cause death and primary composite outcome [3]. In contrast to other large trials, the blood pressure in SPRINT was the mean of three consecutive measurements taken with an automated measuring device in the presence of medical staff. In other words, the procedure was a variation of an "attended" blood pressure measurement, in which a healthcare professional remained in the room while the blood pressure was automatically taken by the device [3-5]. Although the 2018 European guidelines did not specifically refer to the issue of attended vs unattended automatic BP measurement, they did however put forward a recommendation for the use of a validation protocol for the blood pressure measuring device [1]. It remains unresolved if specific validation is needed for patients with CKD $[1,6]$.

We postulated that the difference in blood pressure values between attended and unattended measurements could be larger in patients with CKD due to the different and more complex pathophysiology of AH in patients with CKD that encompasses the excessive activation of the renin-angiotensin-aldosterone system (RAAS), impaired renal sodium excretion, increased peripheral resistance, expansion of extracellular fluid volume and endothelial dysfunction [6, 7]. In patients with CKD, the excessive activation of RAAS is mainly a consequence of glomerular hyperfiltration and hyperperfusion [...]. It can be hypothesised that the impairment of blood pressure regulating systems in patients with CKD could augment the increase of blood pressure during attended measurements of BP.

\section{Objectives}

The aim of the study was to compare the differences in $A B P$ and NABP in patients with different stages of CKD and to assess the effect of concomitant conditions such as diabetes on the differences in $A B P$ and NABP values. 


\section{Material and methods}

\section{Study design and settings}

The study was designed as cross sectional. The measurements began in June 2018 and were completed in February 2019.

\section{Participants}

30 three men and 27 women (mean age $65.6 \pm 14.0$ years and eGFR $41.6 \pm 28.5 \mathrm{ml} / \mathrm{min}$ ) hospitalised either in nephrology or general internal medicine wards were included. 16 patients were classified as CKD stage 1 or 2 with the eGFR higher than $60 \mathrm{ml} / \mathrm{min}, 18$ participants as CKD stage 3, and 26 patients as CKD stage 4 or 5 (eGFR $<30 \mathrm{ml} / \mathrm{min}$ ). 25 patients had been previously diagnosed with type 2 diabetes and were treated with dietary and oral antidiabetic drugs (OADs).

\section{Variables}

The inclusion criteria were as follows: age $>18$ years, previously diagnosed arterial hypertension defined as blood pressure $\geq 140 / 90 \mathrm{~mm} \mathrm{Hg}$ treated with antihypertensive drugs without any changes in antihypertensive therapy in the last 7 days, chronic kidney disease stage 1 to 5 . The exclusion criteria included: unstable blood pressure due to hypotension or systolic blood pressure higher than $160 \mathrm{~mm} \mathrm{Hg}$, chronic renal replacement therapy, history of medication non-compliance, dementia or depression.

\section{Ethical consideration}

The study protocol was approved by the Bioethics Committee of the Medical University of Lodz (Institutional review board approval No. RNN/195/18), and the study was performed according to the Declaration of Helsinki. Written informed consent for participation in the study was granted by all participants.

Blood pressure measurements were taken in the patient room. Blood pressure was first measured via a conventional auscultatory method by a medical staff member. All attended measurements were taken by well-trained and experienced medical personnel, and the cuff was placed on the same arm and location each day, and the conditions of the measurements were kept strictly the same. The measurements were taken after a 5-minute rest in a sitting position in the morning on the first day after admission to hospital. After a 5-minute rest in a sitting position, three additional automated measurements with the OMRON M10-IT device were taken without the presence of medical staff. The device was programmed to delay the start of the series of BP measurements by 5 minutes. After providing the instructions to the patient about how the blood pressure would be measured, a member of the medical staff pushed the START button and left the patient room for the next 15 minutes. The same procedure was repeated over two consecutive days.

\section{Sample size}

Power analysis was used to estimate a sufficient sample size for the study. Since a large previous study revealed a difference of $16 \mathrm{~mm} \mathrm{Hg}$ between attended and unattended BP measured in similar circumstances [8], we expected a difference of $15 \mathrm{~mm}$ $\mathrm{Hg}$ between the measurements. With such assumption, a sample size of 60 will have $95 \%$ power to detect the difference by a paired $t$-test, assuming that the common SD is $10 \%$ with an $\alpha$-significance level of 0.01 .

\section{Statistical methods}

The difference between attended and unattended blood pressure was regarded as the primary endpoint of the study. The results are presented as mean \pm standard deviation (SD) in the case of normally distributed variables and a median with an interquartile range (IQR) for non-normally distributed variables. Non-normally distributed variables, e.g. eGFR, were log transformed for further analysis. A value of $p<0.05$ was regarded as statistically significant. The paired $t$-test or Wilcoxon test was used to compare the attended and unattended blood pressure values, depending on the normality of each variable distribution. The unpaired $t$-test or Mann-Whitney test was used to compare the subgroups of patients with or without diabetes and smokers vs non-smokers. The Pearson's or Spearman's correlation was used to analyse the associations between numerical variables.

\section{Results}

\section{Main results}

All attended blood pressure values were significantly higher than unattended blood pressure values. Significant differences were seen in the case of $\operatorname{SBP}(p=0.009), \operatorname{DBP}(p=0.04)$ and MAP $(p=0.01)$ (Figure 1).

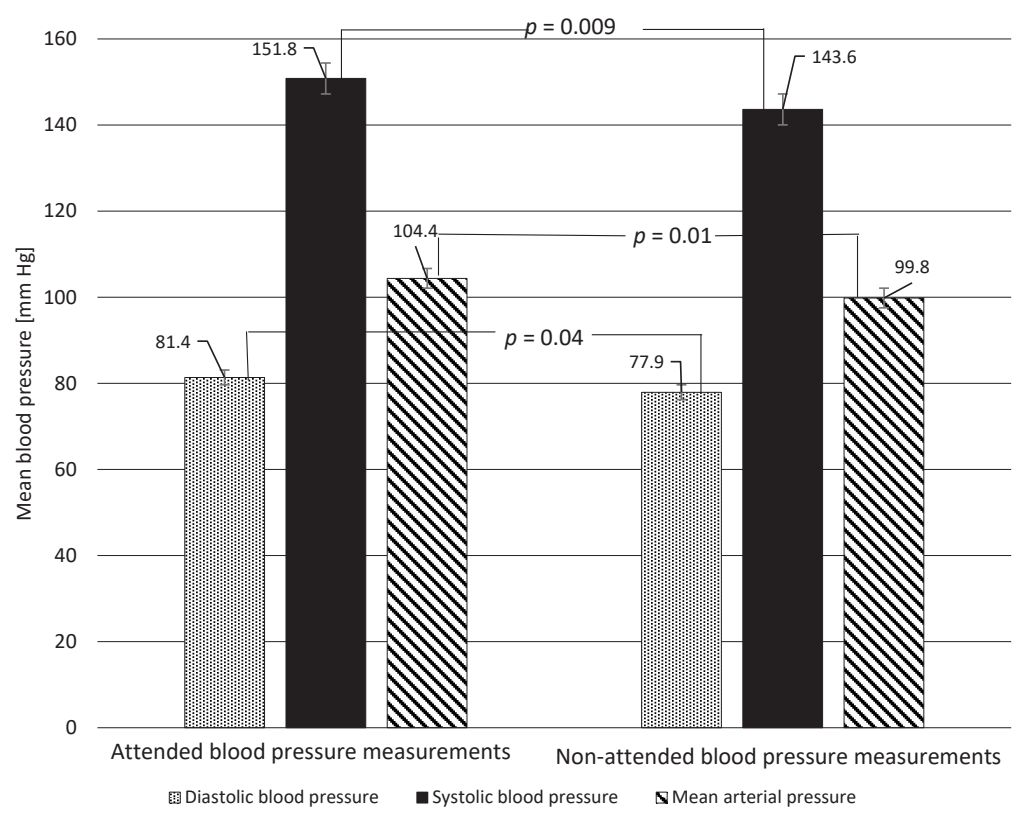

Figure 1. Mean attended and unattended blood pressure values 

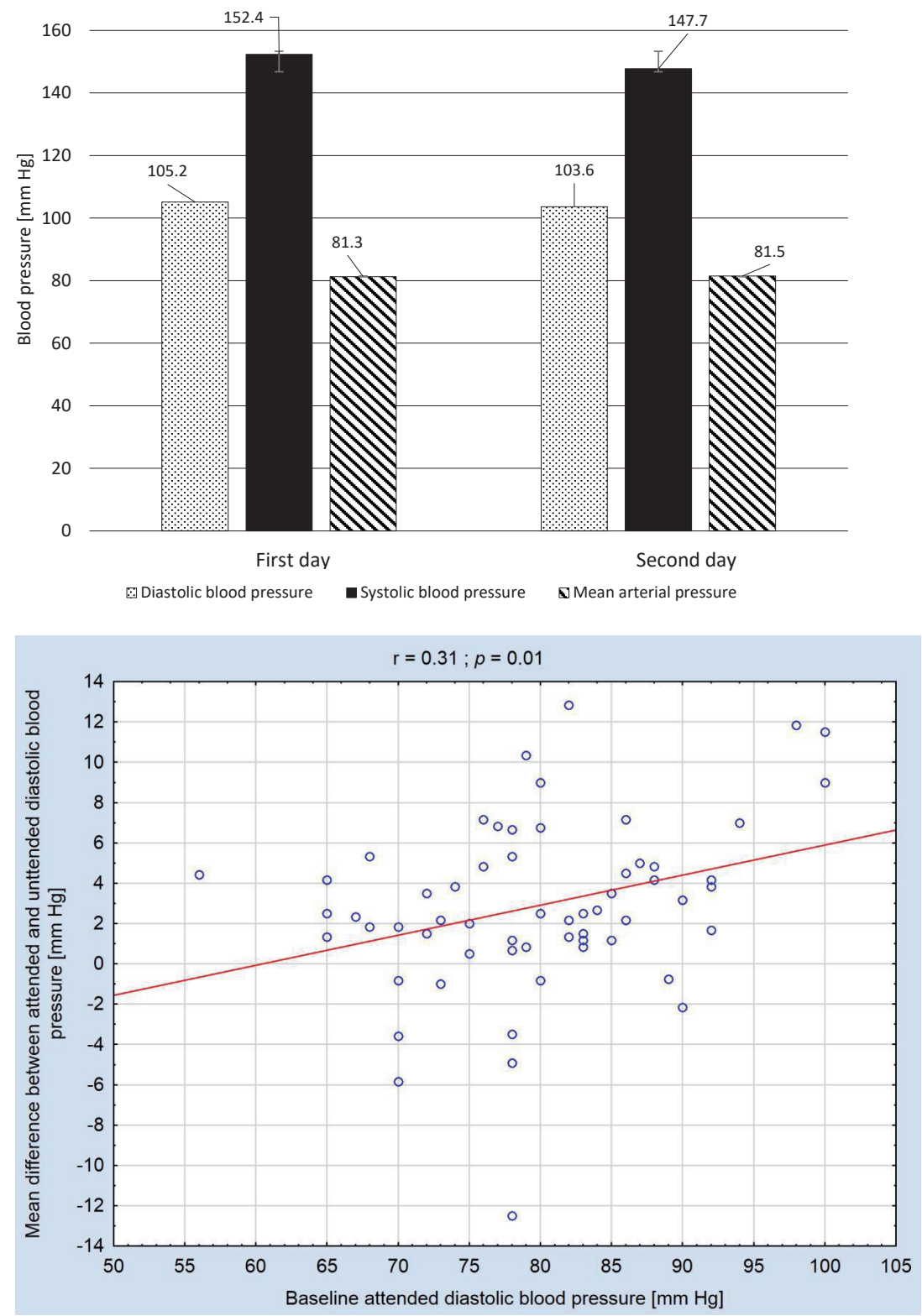

Figure 2. Mean values of blood pressure over two consecutives days
Figure 3. Correlation between the baseline attended diastolic blood pressure and the mean difference between attended and unattended diastolic blood pressure
The differences of blood pressure in unattended measurements taken over two consecutive days were not statistically significant in the case of $\operatorname{SBP}(p=0.08), \operatorname{DBP}(p=0.06)$ and MAP $(p=0.1)$ (Figure 2).

\section{Outcome data}

We did not observed any statistically significant differences between attended and unattended blood pressure values in patients with and without diabetes mellitus and smoking vs non-smoking patients. The median of the study population for eGFR was $33.15 \mathrm{ml} / \mathrm{min}$, and for further analysis, the patients were divided in two groups, i.e. with eGFR lower and higher than the median. There was no statistically significant correlation between attended and unattended blood pressure in both groups (SBP $p=0.9$ and DBP $p=0.07$ ). The correlation between the logarithmic values of eGFR and the differences between attended and unattended blood pressure values was also not statistically significant. No statistically significant correlation was found between the baseline attended systolic blood pressure and the mean difference between attended and unattended systolic blood pressure $(r=0.3, p=0.79)$. There was a statistically significant positive correlation between the baseline attended diastolic blood pressure and the difference between attended and unattended diastolic blood pressure $(r=0.31, p=0.01)$ (Figure 3$)$.

\section{Discussion}

\section{Key results}

Our study showed the significant and potentially clinically relevant differences between attended and unattended blood pressure values in patients with chronic kidney disease regardless of comorbid conditions, including diabetes, severity of renal function impairment and smoking status.

\section{Interpretation}

The study included patients with a wide range of kidney function, from mildly impaired glomerular filtration to advanced CKD. The results of our study are difficult to compare with other studies, since there has only been scarce information on the differences between attended and unattended BP values in patients with high cardiovascular risk, e.g. severe CKD and multiple comorbidities. Furthermore, our results cannot be directly interpreted in the context of the results of SPRINT, due to the inclusion in this trial of only subjects with eGFR in a range of 20-60 $\mathrm{ml} / \mathrm{min}$ and the exclusion of diabetic patients [5, 9]. Other previously published studies with a similar design included patients with different clinical characteristics. In the study that investigated the influence on the blood pressure measurement 
method on blood pressure values performed by Myers et al., the population consisted only of participants with previously diagnosed arterial hypertension but without other major comorbidities [10]. In the study of Bauer et al., there were 45 diabetic participants [4], and in the study of Filipovsky et al., there were 97 diabetic patients [8].

Considering the different pathophysiology of arterial hypertension in patients with and without CKD, it would be interesting to learn whether a technique of blood pressure measurement in patients with CKD would need to be modified in daily practice to avoid a significant white coat effect. The study of Parati et al. confirmed that patients with CKD are at high risk of the development of a white coat effect [11]. However, to our best knowledge, the differences between attended and unattended blood pressure values in diabetic vs non-diabetic patients or patients with different degrees of renal function impairment have not been thoroughly studied. Another important aspect of different blood pressure measurement techniques is the distinctive interpretation of systolic and diagnostic values. Bauer et al. found that only the difference in systolic blood pressure was dependent upon the measurement technique, but the difference in diastolic blood pressure was not statistically significant [4]. Our findings were different, since we were able to reveal the significant differences between $A B P$ and NABP in the case of both systolic and diastolic BP. It is, however, of note that we could not find any relation between the degree of renal function impairment and the difference between attended and unattended BP. This finding is somewhat unexpected, since previous studies found that white coat hypertension is particularly prevalent among patients with CKD [11]. It is expected that the difference between attended and unattended BP could be mostly accounted for by the presence of the medical staff member who takes the measurement, and thus may reflect the white coat reaction [10].

\section{Limitations of the study}

Limitations include the small size of the reference group and the lack of a group of normotensive patients with CKD and hypertensive patients without CKD. Another limitation is the inclusion of patients with a wide spectrum of renal function impairment that resulted in a lack of subgroup analysis. The strength of our study was that the measurements were performed twice over two consecutive days and that we were also able to analyse the difference between the first and the second recording.

\section{Generalizability}

In conclusion, our findings showed statistically significant differences between attended and unattended blood pressure values in a population of patients with high cardiovascular risk. The differences were not significantly affected by the severity of CKD and diabetic or smoking status.

\section{Conclusions}

Our study results allow for the recommendation of a routine use of automated unattended blood pressure measurement in patients with chronic kidney disease regardless of the stage of the disease.

Source of funding: This work was funded by the Medical University of Lodz (Grant No. 503/1-151-02/503-01).

Conflicts of interest: The authors declare no conflicts of interest.

\section{References}

1. Williams B, Mancia G, Spiering W, et al. ESC/ESH guidelines for the management of arterial hypertension. Eur Heart J 2018; 39: 3021-3104 .

2. Arnett DK, Blumenthal RS, Albert MA, et al. ACC/AHA guideline on the primary prevention of cardiovascular disease. J Am Coll Cardiol 2019; 26029 .

3. Cheung AK, Rahman M, Reboussin DM, et al. Effects of intensive BP control in CKD. J Am Soc Nephrol 2017; 28: $2812-2823$.

4. Bauer F, Seibert FS, Rohn B, et al. Attended versus unattended blood pressure measurements in a real life setting. Hypertension 2018; 71: 243-249.

5. Agarwal R. Implications of blood pressure measurement technique for implementation of Systolic Blood Pressure Intervention Trial (SPRINT). J Am Heart Assoc 2017; 6: e004536, doi: 10.1161/JAHA.116.004536.

6. Cushman WC, Whelton PK, Fine LJ, et al. SPRINT trial results latest news in hypertension management. Hypertension 2016; 67: 263-265 .

7. Judd E, Calhoun DA. Management of hypertension in CKD: beyond the guidelines. Adv Chronic Kidney Dis 2015; 22(2): 116-122, doi: 10.1053/j.ackd.2014.12.001.

8. Filipovský J, Seidlerová J, Ceral J, et al. A multicentre study on unattended automated office blood pressure measurement in treated hypertensive patients. Blood Press 2018; 27: 188-193.

9. Myers MG. The great myth of office blood pressure measurement. J Hypertens 2012; 30: 1894-1898.

10. Myers MG, Valdivieso MA, Kiss A. Use of an automated blood pressure recording device, the BpTRU, to reduce the "white coat effect" in routine practice. Am J Hypertens 2003; 16: 494-497.

11. Parati G, Ochoa JE, Bilo G. White coat and masked hypertension in chronic kidney disease: importance of the difference between office and out-of-office blood pressure measurements. J Am Heart Assoc 2019; 8, doi: 10.1161/JAHA.119.012299.

Tables: 0

Figures: 3

References: 11

Received: 3.06 .2019

Reviewed: 27.06 .2019

Accepted: 12.08 .2019

Address for correspondence:

Prof. Michał Nowicki

Klinika Nefrologii, Hipertensjologii i Transplantologii Nerek

Centralny Szpital Kliniczny Uniwersytetu Medycznego w Łodzi

ul. Pomorska 251, 92-213 Lódź, Polska

Tel.: +48 42 201-44-00, e-mail: nefro@wp.pl 